\section{Associação entre condições socioeconômicas, sanitárias e de atenção básica e a morbidade hospitalar por doenças de veiculação hídrica no Brasil}

\author{
Association between socioeconomic, health, and \\ primary care conditions and hospital morbidity \\ due to waterborne diseases in Brazil
}

\section{Asociación entre condiciones socioeconómicas, sanitarias y de atención básica y la morbilidad hospitalaria por enfermedades de transmisión hídrica en Brasil}

Roberta Fernanda da Paz de Souza Paiva 1 Marcela Fernanda da Paz de Souza 2

\section{Resumo}

O presente estudo teve como objetivo avaliar a associação entre condições socioeconômicas, de saneamento básico e de cobertura por equipes de saúde da família $(E q S F)$ sobre as internações por doenças de veiculação hídrica no Brasil. A análise das condições socioeconômicas e de saneamento se deu por meio de um estudo ecológico com dados da Pesquisa Nacional por Amostra de Domicílios (PNAD) e do Departamento de Informática do SUS (DATASUS) - observações dos Estados e Distrito Federal - para o ano de 2013. A relação das EqSF com as internações foi avaliada por meio da estimação de regressão simples, com dados coletados no DATASUS e Departamento de Atenção Básica do Ministério da Saúde, para o período de 1998 a 2014. A existência de coleta de esgoto por rede geral, a baixa escolaridade e a cobertura por EqSF influenciaram, com significância estatística $(p<0,05)$, as internações avaliadas. Pela análise do risco atribuível, estimou-se que no Brasil, tomando-se como base os dados de 2013, 57.574 (16,3\%) das internações por doenças selecionadas poderiam ter sido evitadas, caso as condições de esgotamento sanitário fossem adequadas. Seriam ainda evitados os gastos com o tratamento delas, calculado em $R \$ 20.372 .559,90$ e os dias perdidos com as internações, 172.722. Sugerese que a adoção integrada de políticas de saneamento, educação e assistência à saúde que considerem as desigualdades regionais contribuirá para a melhoria das condições de saúde da população.

Poluição Ambiental; Saneamento Básico; Doenças Transmitidas pela Água; Assistência à Saúde

\author{
Correspondência \\ R. F. P. S. Paiva \\ Universidade Federal Fluminense. \\ Av. dos Trabalhadores 420, Volta Redonda, RJ \\ 27255-125, Brasil. \\ robertapaz2003@yahoo.com.br \\ 1 Universidade Federal Fluminense, Volta Redonda, Brasil. \\ 2 Universidade Federal do Rio Grande do Norte, Natal, Brasil.
}




\section{Introdução}

Os elevados níveis de poluição ambiental observados atualmente vêm acarretando perdas econômicas e de bem-estar da população ${ }^{1}$, que se torna cada vez mais exposta ao acometimento de doenças relacionadas à variações na quantidade e na qualidade dos recursos naturais disponíveis 2 . Nesse contexto, a contaminação hídrica é um dos principais problemas ambientais enfrentados pela população, estando diretamente ligada à perda das condições de saúde dos indivíduos, especialmente nos grupos mais vulneráveis e regiões mais pobres 2,3.

A falta de saneamento ambiental adequado é tida como uma das principais causas da poluição e da contaminação das águas para o abastecimento humano e está, portanto, contribuindo para os casos de doenças de veiculação hídrica 4,5,6,7,8,9,10.

No Brasil, as condições de saneamento ambiental, apesar de apresentarem melhoras nos últimos anos, ainda são deficientes. Segundo dados da Pesquisa Nacional de Saneamento Básico 11, apenas 55\% dos municípios brasileiros possuíam, em 2008, rede coletora de esgoto e, do total do esgoto coletado, apenas $68,8 \%$ passavam por algum tipo de tratamento antes de ser depositados nos corpos dos rios. Outros dados importantes da pesquisa indicam que até o referido ano o destino dos resíduos sólidos em 50,8\% dos municípios eram os "lixões" e que em 12,8\% dos municípios a água fornecida era apenas parcialmente tratada ou não passava por nenhum tipo de tratamento $(6,6 \%)$.

Tal realidade não se verifica apenas no Brasil. Segundo dados da Organização Mundial da Saúde (OMS) 12, em 2011, 64\% da população mundial não contavam com instalações sanitárias melhoradas e 89\% usavam água potável proveniente de uma fonte adequada, além de outras deficiências no sistema de saneamento.

Melhorias na qualidade da água, no saneamento básico e nas condições de higiene da população poderiam reduzir os casos de doenças, sobretudo em grupos etários mais vulneráveis, como crianças e idosos 2. Estudos já realizados comprovaram a associação entre saneamento inadequado e casos de diarreia no Brasil 13,14,15,16,17 e em outros países, como a África do Sul 18 e países da América Latina 19, tendo em conta, na maioria dos casos, crianças e idosos.

Outras doenças de veiculação hídrica atingem grupos etários distintos e também são responsáveis, juntamente com a diarreia, por elevado número de internações. No ano de 2015, segundo dados do Departamento de Informática do SUS (DATASUS. Informações de saúde. http://www2.datasus.gov. br/DATASUS/index.php, acessado em 10/Mar/2015), doenças como cólera, febres tifoide e paratifoide, shiguelose, amebíase, diarreia e gastroenterite de origem infecciosa presumível, esquistossomose e outras doenças infecciosas intestinais foram responsáveis por 2,35\% das internações totais no Brasil, gerando uma parcela de 0,7\% dos gastos totais do Sistema Único de Saúde (SUS) com internações no período. Dessas internações, 43,4\% foram de pessoas com 10 anos ou mais de idade.

Quando considerada a distribuição regional desses casos, tem-se que a maior concentração das internações ocorreu na Região Nordeste, onde foram registrados 49,1\%. Nessa região, quando comparada às demais, encontrou-se a menor renda média mensal, cerca de 55,4\% da renda média mensal da Região Centro-oeste, a que apresentou melhor resultado para o indicador no ano de 2013, segundo dados do Instituto Brasileiro de Geografia e Estatística (IBGE. Pesquisa Nacional por Amostra de Domicílios - 2013. http://www.sidra.ibge.gov.br/pnad/, acessado em 29/Jun/2016). Além da menor renda, encontrou-se na citada região a menor taxa de alfabetização entre grandes regiões brasileiras: $83,5 \%$.

Outro fator importante a ser considerado na ocorrência dessas internações é a atenção básica, já que algumas doenças de veiculação hídrica encontram-se na lista brasileira de condições sensíveis à atenção primária (CSAP). Diversos estudos já encontraram associação entre a atenção primária e internações infantis no Brasil por CSAP 20,21,22 e internações por CSAP em outros grupos etários no Brasil e por localidades específicas 23,24,25.

Segundo dados do DATASUS (Indicadores de morbidade. http://tabnet2.datasus.gov.br/cgi/def tohtm.exe?idb2013/d32.def, acessado em 10/Jan/2017), em 2013, as taxas de internação (número de casos por 10 mil habitantes) por casos de gastroenterites infecciosas e complicações (grupo 2 das CSAP) nas regiões Norte $(37,17)$ e Nordeste $(35,44)$ foram maiores que os demais grupos da CSAP nessas regiões e superiores à média nacional $(19,94)$. Os casos representaram ainda grande parte das internações totais por CSAP nas regiões Norte e Nordeste, sendo iguais a 26,6\% e 28,15\%, respectivamente. 
Diante do exposto, buscou-se neste trabalho avaliar a associação entre condições de saneamento, assistência básica de saúde e variáveis socioeconômicas às internações pelo grupo de doenças selecionadas que, segundo a literatura, estariam relacionadas à deficiência nos serviços de saneamento disponíveis. Pretendeu-se considerar nas análises grupos etários e de doenças que habitualmente não constam dos estudos realizados, além de captar o custo econômico associado às internações atribuídas ao grupo de doenças no período considerado. De forma descritiva, com intuito de identificar as regiões com maior ocorrência dos casos, tratou-se ainda de calcular indicadores de saúde para as doenças de veiculação hídrica para o Brasil e por grandes regiões.

\section{Métodos}

Trata-se de um estudo ecológico no qual foram utilizados dados para o Brasil, sendo as unidades de análise compostas pelos 26 estados brasileiros e o Distrito Federal.

A análise da associação das condições de saneamento básico e socioeconômicas da população com os casos de internações por doenças veiculadas por poluição hídrica baseou-se na estimação de uma função dose-resposta, por uma regressão linear múltipla 26 . Nessa regressão, foi testada a associação entre as variáveis independentes (aquelas relacionadas ao saneamento básico e socioeconômicas) e a variável dependente selecionada (internações por doenças de veiculação hídrica).

As variáveis consideradas no modelo foram as seguintes:

- Variável dependente: internação hospitalar - número de internações por doenças selecionadas segundo lugar de residência para o ano de 2013. As doenças consideradas foram selecionadas por estarem relacionadas à transmissão direta pela água, por meio da ingestão ou contato (ou penetração na pele) com ela 5, a saber: cólera, febres tifoide e paratifoide, shiguelose, amebíase, diarreia e gastroenterite de origem infecciosa presumível, outras doenças infecciosas intestinais, esquistossomose (Classificação Internacional de Doenças, 10a revisão - CID-10: A00-A09; B65).

- Variáveis explicativas: (i) socioeconômicas - população (número de habitantes), renda média (valor do rendimento médio mensal das pessoas de 10 anos ou mais em Reais), escolaridade (população com 10 anos ou mais de idade sem instrução ou com menos de 1 ano de escolaridade); (ii) relacionadas ao saneamento básico: abastecimento (número de moradores em domicílios que possuem abastecimento de água por rede geral), esgoto (número de moradores em domicílios que possuem esgotamento sanitário por rede geral).

O modelo foi estimado com todas as variáveis, sendo selecionadas, ao final da estimação, aquelas com significância estatística menor ou igual a $5 \%(\mathrm{p}<0,05)$. Com base nos resultados da regressão, foi estimada uma regressão restrita, ou seja, com as variáveis consideradas significativas na primeira estimação. Como a variável dependente apresentou distribuição não normal, foi utilizada na estimação sua transformação logarítmica, que apresentou distribuição normal.

A colinearidade foi avaliada por fator de inflação da variância (FIV), em que valores inferiores a 10 indicam a ausência de problemas de colinearidade 26 . As variáveis população e abastecimento foram excluídas da regressão múltipla por apresentarem problemas de colinearidade. Quando realizada a regressão linear simples, ambas as variáveis se apresentaram significativas e correlacionadas com a variável independente. As análises foram realizadas com o software IBM SPSS, versão 20.0 (IBM Corp., Armonk, Estados Unidos).

Após a estimação da regressão múltipla, procedeu-se ao cálculo do número de casos e o custo de internações associados às condições de saneamento consideradas estatisticamente significativas na estimação, sendo calculada a fração de risco atribuível.

Segundo Wagner \& Callegari-Jacques 27, o risco atribuível e suas frações são medidas de associação que permitem avaliar os impactos da exposição à variável independente sobre as internações consideradas no estudo. Possuem uma perspectiva de saúde pública e de planejamento de saúde, pois permitem que sejam identificados os impactos de um fator de risco sobre uma população e os principais impactos de sua remoção.

O número de internações atribuíveis foi calculado com base no risco relativo (RR), segundo PrüssÜstün et al. 28 e Rodrigues et al. 29 :

$$
\text { Número de internações atribuíveis }=\mathrm{I} z, \mathrm{t} * \text { FA }
$$


Onde I z,t é o número de internações associadas à causa $z$, no tempo t (no caso o ano de 2013) e FA a fração atribuível, que teve seu cálculo baseado no RR calculado a pelos resultados da regressão múltipla, sendo:

O RR pode ser dado por 30,31: $\quad F A=(R R-1) / R R$

$$
\mathrm{RR}=e \beta \chi
$$

Onde $\beta$ é o parâmetro estimado pela equação e $\chi$ a variação interquartil (VIQ) na variável considerada para o cálculo.

Para que se pudesse avaliar a associação entre as doenças selecionadas e os programas de assistência à saúde (neste caso o Programa Saúde da Família), procedeu-se à estimação de uma regressão linear simples, que teve como variável dependente o número de internações por doenças selecionadas, e, como variável independente, a cobertura estimada por equipes de saúde da família (EqSF). Nesse caso, por entender-se que os resultados do trabalho executado por essas equipes são observados ao longo do tempo, tratou-se de avaliar a associação para os resultados do Brasil para o período de 1998 a 2014.

No caso dos indicadores de saúde, foram analisados dados para o Brasil e grandes regiões (Norte, Nordeste, Sudeste, Sul, Centro-oeste), sendo considerado, além do grupo de doenças selecionadas, o grupo de doenças infectoparasitárias - DIP (CID-10, capítulo I: A00-B99).

Foram calculados indicadores de morbidade, do comprometimento da rede de serviços de saúde e dos gastos com as internações para o Brasil e grandes regiões no ano de 2013, adotando-se os procedimentos propostos pela Fundação Nacional de Saúde (Funasa) 5, sendo:

Taxa de internação hospitalar por DIP (por grande região) = número de internações por DIP (por grande região)/população (por grande região) * 100.000

Taxa de internação hospitalar por doenças selecionadas = número de internações por doenças selecionadas (por grande região)/população (por grande região) * 100.000

Proporção de internações por DIP (por grande região) = número de internações por DIP (por grande região)/número de internações por todas as causas (por grande região) * 100

Proporção de internações por doenças selecionadas (por grande região) = número de internações por doenças selecionadas (por grande região)/número de internações por todas as causas (por grande região) * 100

Proporção de gastos hospitalares por DIP (por grande região) = gastos de internação por DIP (por grande região)/gasto total de internações hospitalares (por grande região)

Proporção de gastos hospitalares por doenças selecionadas (por grande região) = gastos de internação por doenças selecionadas (por grande região)/gasto total de internações hospitalares (por grande região)

\section{Fonte de dados}

Os dados utilizados são de base secundária. As informações sobre as internações, valores de internações, dias de internações e outras referentes à morbidade foram obtidas no DATASUS (http:// www.datasus.gov.br); os dados acerca das condições de saneamento básico (esgoto e abastecimento) e socioeconômicas (população, renda e escolaridade) foram obtidos na Pesquisa Nacional por Amostra de Domicílios - PNAD (http://www.sidra.ibge.gov.br); as demais informações referentes à assistência básica foram coletadas no Departamento de Atenção Básica (DAB), Secretaria de Atenção à Saúde, Ministério da Saúde. 


\section{Resultados}

No modelo de regressão múltipla, ao se avaliar a associação entre as internações e as variáveis de saneamento e socioeconômicas, verificou-se que as variáveis relacionadas com significância estatística $(\mathrm{p}<0,05)$ foram a cobertura por coleta de esgoto por rede geral no domicílio e a escolaridade da população (Tabela 1).

Os resultados da regressão indicaram boa correlação entre as variáveis explicativas e a variável dependente, com R2 de 0,67. Os testes de colinearidade (tolerância e FIV) indicam que não há problemas que possam prejudicar as análises.

Para os dados considerados, entende-se que residir em um domicílio com cobertura por esgotamento sanitário por rede geral se relaciona inversamente proporcional às internações por doenças de veiculação hídrica, sugerindo que melhorias nas condições sanitárias da população poderiam acarretar em redução do número de internações pelas referidas doenças.

Pelo coeficiente estimado na regressão múltipla, pode-se calcular o número de casos e o custo associados às doenças selecionadas atribuíveis ao esgotamento por rede de coleta geral. Seguindo o método proposto (equações 1, 2 e 3), calculou-se o RR de 0,86 e uma fração atribuível de 16,3\%, que indica uma redução no risco de internação por doenças veiculadas pela água devido à elevação do número de indivíduos residentes em domicílios que possuem coleta de esgoto por rede geral.

O cálculo do número de internações atribuíveis às doenças selecionadas indicou que, no Brasil, tomando-se como base os dados de 2013, 57.574 (cerca de 16,3\%) das internações por doenças selecionadas (353.666) poderiam ter sido evitadas, caso as condições de esgotamento sanitário fossem adequadas. Seriam ainda evitados os gastos com o tratamento delas, que no caso deste estudo calculou-se em $\mathrm{R} \$ 20.372 .559,90$ (dado valor médio dessas internações de $\mathrm{R} \$ 353,85$ ) e os dias perdidos com as internações, 172.722 (dada permanência média por internação de 3 dias).

Os níveis de escolaridade também apresentaram significância estatística na determinação das internações, apresentando relação direta entre baixa escolaridade e as internações consideradas.

Destaca-se aqui que as variáveis excluídas do modelo ou que não apresentaram significância estatística, também contribuem para as internações analisadas, conforme comprovam as regressões simples realizadas e a literatura. Entretanto, a regressão não foi capaz de captar essa associação quando avaliadas em conjunto com as demais.

A associação entre a atenção básica e as internações pelas doenças selecionadas foi avaliada mediante estimação de regressão linear simples (Tabela 2).

A cobertura de atendimento pelas EqSF mostra relação inversamente proporcional à morbidade hospitalar no período estudado, indicando que maior cobertura por EqSF deve levar à redução do número de casos de internação pelas doenças consideradas no estudo.

O cálculo dos indicadores de saúde mostra que as internações por doenças veiculadas pela água ocorrem no Brasil todo. No entanto, nas regiões Norte e Nordeste, apresentam maiores proporções (Tabela 3).

Tabela 1

Resultados para regressão linear múltipla restrita entre as internações por doenças associadas à poluição hídrica, escolaridade e domicílios com coleta de esgoto por rede geral, por Unidade da Federação, Brasil, 2013.

\begin{tabular}{|c|c|c|c|c|}
\hline \multirow[t]{2}{*}{ Variável } & \multirow[t]{2}{*}{ Coeficiente não padronizado } & \multirow[t]{2}{*}{ Coeficiente padronizado } & \multicolumn{2}{|c|}{ Colinearidade } \\
\hline & & & Tolerância & FIV \\
\hline Escolaridade & 0,001 & 1,219 * & 0,400 & 2,501 \\
\hline Domicílios com coleta de esgoto por rede geral & $-0,0000473$ & $-0,663$ * & 0,400 & 2,501 \\
\hline
\end{tabular}

FIV: fator de inflação da variância.

Nota: $\mathrm{R}^{2}=0,67$.

* Significante a 0,001. 
Tabela 2

Resultados para regressão linear simples entre as internações por doenças associadas à poluição hídrica e a cobertura por equipes de saúde da família (EqSF). Brasil, 1998 a 2014.

\begin{tabular}{lccc}
\hline Variável & Coeficiente não padronizado & Coeficiente padronizado & $\begin{array}{c}\text { Colinearidade } \\
\text { FIV }\end{array}$ \\
\hline Cobertura por EqSF & $-3787,9$ & $-0,708$ & 1,000 \\
\hline
\end{tabular}

FIV: fator de inflação da variância.

Nota: $\mathrm{R}^{2}=0,501$.

Tabela 3

Indicadores de saúde para doenças infectoparasitárias (DIP) e doenças relacionadas à poluição hídrica. Brasil e grandes regiões, 2013.

\begin{tabular}{|c|c|c|c|c|c|c|}
\hline & \multicolumn{3}{|c|}{ DIP } & \multicolumn{3}{|c|}{ Doenças relacionadas à poluição hídrica } \\
\hline & $\begin{array}{c}\text { Taxa de } \\
\text { internação }\end{array}$ & $\begin{array}{l}\text { Proporção de } \\
\text { internações }\end{array}$ & $\begin{array}{c}\text { Proporção de } \\
\text { gastos }\end{array}$ & $\begin{array}{c}\text { Taxa de } \\
\text { internação }\end{array}$ & $\begin{array}{l}\text { Proporção de } \\
\text { internações }\end{array}$ & $\begin{array}{l}\text { Proporção de } \\
\text { gastos }\end{array}$ \\
\hline Brasil & 430,38 & 7,74 & 6,82 & 175,55 & 3,15 & 0,99 \\
\hline Norte & 690,32 & 11,67 & 8,93 & 358,09 & 6,05 & 2,88 \\
\hline Nordeste & 623,28 & 11,40 & 8,00 & 337,88 & 6,18 & 2,23 \\
\hline Sul & 353,11 & 5,34 & 5,21 & 114,45 & 1,73 & 0,48 \\
\hline Sudeste & 275,74 & 5,37 & 6,82 & 56,08 & 1,09 & 0,34 \\
\hline Centro-oeste & 437,72 & 7,43 & 5,82 & 155,12 & 2,63 & 0,88 \\
\hline
\end{tabular}

Fonte: elaborado a partir de DATASUS (Informações de saúde. http://www2.datasus.gov.br/DATASUS/index.php, acessado em 10/Mar/2015).

Na Região Norte, a taxa de internação por doenças veiculadas pela água no período foi de 358,09 casos por 100 mil habitantes, mais que o dobro da média nacional de 175,55. No Nordeste, também se encontrou uma taxa de internação elevada, de 337,88 casos por 100 mil habitantes.

As proporções dessas internações nas regiões Norte e Nordeste indicam que o número de internações por doenças associadas à poluição hídrica respondeu por cerca de $6 \%$ das hospitalizações totais nessas regiões. É um valor considerado elevado quando comparado à média nacional (3,2\%) e ainda quando se entende que muitos desses casos poderiam ser evitados com a adoção de medidas que contribuíssem com a melhoria das condições de vida da população. Observa-se ainda que, nessas duas regiões, tais internações e seus gastos são parte significativa de todas as internações e gastos com o grupo de todas as doenças infectoparasitárias.

Quando são analisados os dados dessas duas regiões desagregados por estados, tem-se que apenas cerca de $42 \%$ dos estados da Região Norte e $22 \%$ dos estados da Região Nordeste apresentam proporção de internações pelas doenças selecionadas em relação ao total menor que a média nacional (Tabela 4).

Na Região Norte, os estados de Pará (8,68\%), Acre (5,45\%), Rondônia (4,06\%) e Tocantins (3,35\%) apresentam a proporção de internação por doenças relacionadas à água acima da média nacional (3,2\%), estando no Estado do Pará o caso mais crítico, já que 8,68\% das internações são pelas referidas doenças e o gasto com elas (4,32\%) se apresenta 4 vezes maior que a média nacional $(0,99 \%)$.

$\mathrm{Na}$ Região Nordeste, a situação é ainda pior, visto que em apenas 2 dos seus 9 estados a proporção de internações por doenças associadas à poluição da água é menor que a média nacional (Pernambuco e Sergipe). No Estado do Maranhão, caso mais crítico, a proporção é de 11,82\% de internações pelas citadas doenças em relação às demais internações no estado e no Piauí, de 9,08\%. Observa-se ainda que, nesses estados, uma parcela significativa dos gastos com internações do SUS, respectivamente $5,56 \%$ e 4,08\%, foram destinados ao pagamento de internações por casos que poderiam, na sua maioria, ser evitados por meio de medidas preventivas. 


\section{Tabela 4}

Proporção de internações e de gastos por doenças veiculadas pela água selecionadas no estudo em relação ao total de internações e gastos para o Brasil, estados das regiões Norte e Nordeste, 2013.

\begin{tabular}{lcc}
\hline & $\begin{array}{c}\text { Proporção (\%) de internações por } \\
\text { doenças veiculadas pela água } \\
\text { selecionadas }\end{array}$ & $\begin{array}{c}\text { Proporção (\%) de gastos por doenças } \\
\text { veiculadas pela água selecionadas }\end{array}$ \\
\hline Brasil & 3,2 & 0,99 \\
Região Norte & 4,06 & 1,95 \\
Rondônia & 5,45 & 2,58 \\
Acre & 3,00 & 1,33 \\
Amazonas & 1,91 & 1,26 \\
Roraima & 8,68 & 4,32 \\
Pará & 2,44 & 1,26 \\
Amapá & 3,35 & 1,34 \\
Tocantins & & \\
Região Nordeste & 11,82 & 5,56 \\
Maranhão & 9,08 & 4,08 \\
Piauí & 4,65 & 1,59 \\
Ceará & 6,51 & 1,88 \\
Rio Grande do Norte & 6,54 & 2,16 \\
Paraíba & 2,95 & 0,98 \\
Pernambuco & 5,09 & 1,90 \\
Alagoas & 1,82 & 0,62 \\
Sergipe & 6,05 & 2,31 \\
Bahia & 5,85 & \\
\hline
\end{tabular}

Fonte: elaborado a partir de DATASUS (Informações de saúde. http://www2.datasus.gov.br/DATASUS/index.php,

acessado em 10/Mar/2015).

\section{Discussão}

As estimações econométricas realizadas indicam a relação, com significância estatística, entre as internações por doenças de veiculação hídrica e esgotamento sanitário por rede geral (inversa), baixa escolaridade (direta) e cobertura pelas EqSF (inversa). Para os dados considerados no estudo, tem-se de residir em um domicílio com cobertura por esgotamento sanitário por rede geral, que se relaciona inversamente proporcional às internações por doenças associadas à poluição da água, indicando que melhorias nas condições sanitárias da população poderiam acarretar em redução do número de internações pelas referidas doenças.

Outros trabalhos já encontraram resultados que confirmaram a associação entre esgotamento sanitário/poluição hídrica e morbidade/mortalidade por doenças relacionadas à inadequação dos sistemas de coleta de esgoto no Brasil e também no exterior 32,33. Em muitos desses estudos, concluiuse que a falta de condições de saneamento estaria relacionada de maneira direta à morbidade $14,15 \mathrm{e}$ mortalidade 15,16 por diarreias no Brasil.

Os investimentos em saneamento contribuiriam, além da melhoria nas condições de saúde, para a redução do impacto da pobreza e das desigualdades nas comunidades mais vulneráveis 17 .

Em outro estudo 18, que objetivou avaliar os impactos da poluição da água e do saneamento inadequado na saúde humana (particularmente nos casos de diarreia) nos residentes em habitações de baixo custo na Cidade do Cabo, África do Sul, foram encontrados resultados indicando que as condições de moradia e saneamento, além das condições de higiene, contribuíam para a piora do estado de saúde da população avaliada. 
O nível de escolaridade da população também está associado às internações consideradas, indicando o resultado que a baixa escolaridade dos indivíduos contribui para o aumento da referida morbidade. Esse achado está de acordo com o esperado e já encontrado por outros autores, que avaliaram os determinantes da morbi-mortalidade 15 e morbidade 16 por diarreia e por doenças de veiculação hídrica, respectivamente.

A cobertura por EqSF contribui, segundo os dados, para a redução dos casos de internação. A relação encontrada se justifica, dadas as atribuições dos profissionais que delas fazem parte. $\mathrm{O}$ atendimento da população por essas equipes nas unidades básicas de saúde ou mesmo nos domicílios permite que seja desenvolvido o trabalho de prevenção contra o acometimento das doenças e o tratamento de casos delas, antes que seja necessária a internação do paciente 34 . Sendo esse seu papel fundamental na orientação quanto às condições de higiene e cuidados com a saúde.

Outros trabalhos já comprovaram a associação entre o atendimento por essas equipes e a redução de internações por alguns grupos de doenças 20,21,22,23,24,25, evidenciando a importância da adoção e do reforço, por parte do poder público, de estratégias que tenham como objetivo a promoção, prevenção e recuperação da saúde dos indivíduos.

As trocas de informações técnicas pelas equipes e de saberes e experiências pelos indivíduos atendidos contribui para que haja mudança de comportamentos e a busca de formas saudáveis de vida, tendo a educação popular em saúde um papel fundamental nas EqSF 35

Nesse contexto, a orientação quanto às simples práticas do cotidiano pode melhorar significativamente a vida da população. Estudos realizados no Paquistão 36 e em Bangladesh 37 indicaram a importância da intervenção de profissionais da saúde ou membros da comunidade previamente treinados na redução de casos de diarreias nos anos de 2004 e 2007, respectivamente. As ações das pesquisas constavam, sobretudo, na distribuição de sabão comum e bactericida às populações carentes selecionadas e na orientação delas quanto às práticas de higiene, para posterior análise do comportamento dos casos da doença, práticas que obtiveram resultados positivos. Essa discussão sugere que o trabalho de educação das famílias quanto às práticas de higiene e seus impactos na saúde podem ter papel fundamental na melhoria da qualidade de vida da população.

A análise da distribuição espacial das internações e gastos por doenças veiculadas à água, tendo-se em conta as grandes regiões brasileiras, revela que, nas regiões mais pobres, encontram-se as maiores taxas de internação e proporção de gastos. As regiões Norte e Nordeste concentram grande parte dessas internações no Brasil, sendo as mesmas a apresentar menores níveis de renda média e escolaridade em relação às demais regiões (IBGE. Pesquisa Nacional por Amostra de Domicílios - 2013. http://www.sidra.ibge.gov.br/pnad/, acessado em 29/Jun/2016). Nessas regiões, também existe um elevado déficit de acesso aos serviços de água e esgoto, estando sua participação nos investimentos em sistemas de água e esgoto muito aquém da participação no déficit 38 . Esse resultado é condizente com outras publicações que também destacaram a elevada participação das regiões Norte e Nordeste nos casos de internações por doenças do grupo das DIP e a proporção delas em relação ao total de internações da região 5,17,39. Verifica-se que, em alguns estados, as proporções de internações e gastos com tais internações são mais elevadas, o que demonstra a necessidade de estudos localizados que possam embasar medidas e direcionar recursos que visem reduzir a ocorrência desses casos.

Como principais limitações do trabalho, tem-se o fato de que os estudos ecológicos, tais como os utilizados, podem não captar todas as causas que explicam os casos das internações avaliadas pelo modelo de regressão múltipla, conforme já citado, e a chamada "falácia ecológica", que indica que a associação entre o fator de exposição e o evento pode ocorrer não com o indivíduo, mas sim com a população, pelo fato de considerarem a população e não o indivíduo como unidade de análise. Apesar disso, os estudos ecológicos podem ajudar a identificar fatores que merecem uma investigação mais detalhada com estudo de maior capacidade analítica, além de serem adequados em casos em que haja necessidade de intervenções em nível populacional 40, como é o caso das intervenções discutidas no trabalho.

As internações por doenças veiculadas pela água estão associadas às condições de saneamento básico, escolaridade e cobertura por serviços de atenção básica. Sugere-se que a adoção integrada de políticas de saneamento, a educação e a assistência à saúde, que considerem as desigualdades regionais, contribuirão para a melhoria das condições de saúde da população e dos indicadores de saúde para as referidas doenças. 


\section{Colaboradores}

R. F. P. S. Paiva e M. F. P. Souza participaram de todas as etapas de elaboração do artigo.

\section{Agradecimentos}

À Pró-reitoria de Pesquisa, Pós-graduação e Inovação da Universidade Federal Fluminense (Edital FOPIN 2015) pelo apoio financeiro e à engenheira Paula Cristina Carvalho pelo auxílio na coleta e tabulação dos dados.

\section{Referências}

1. Millennium Ecosystem Assessment. Ecosystems and human well-being: synthesis. Washington DC: Island Press; 2005.

2. Prüss-Üstün A, Bos R, Gore F, Bartram J. Safer water, better health: costs, benefits and sustainability of interventions to protect and promote health. Geneva: World Health Organization; 2008.

3. Ostro B. Outdoor air pollution: assessing the environmental burden of disease at national and local levels. Geneva: World Health Organization; 2004. (Environmental Burden of Disease Series, 5).

4. Heller L. Saneamento e saúde. Brasília: Organização Pan-Americana da Saúde/Organização Mundial da Saúde; 1997.

5. Fundação Nacional de Saúde. Impactos na saúde e no Sistema Único de Saúde decorrentes de agravos relacionados a um saneamento ambiental inadequado. Brasília: Fundação Nacional de Saúde; 2010.

6. Kronenberger DMP, Pereira RS, Freitas EAV, Scarcello JA, Cleverário Junior J. Saneamento e meio ambiente. In: Instituto Brasileiro de Geografia e Estatística. Atlas de saneamento 2011. http://www.ibge.gov.br/home/estatistica/po pulacao/atlas_saneamento/default_zip.shtm (acessado em 10/Mar/2016).

7. Andreazzi MAR, Barcellos C, Hacon S. Velhos indicadores para novos problemas: a relação entre saneamento e saúde. Rev Panam Salud Pública 2007; 22:211-7.

8. Tucci CEM. Águas urbanas. Estud Av 2008; 22:1-16.

9. Ercumen A, Gruber JS, Colford JM. Water distribution system deficiencies and gastrointestinal illness: a systematic review and meta-analysis. Environ Health Perspect 2014; 122:651-60.

10. Tang M, Fan Y, Wang G. Comprehensive costbenefit evaluation for the improvement of rural water supply in Hunan Province. Zhonghua Yu Fang Yi Xue Za Zhi 1996; 30:23-25.
11. Instituto Brasileiro de Geografia e Estatística. Pesquisa Nacional de Saneamento Básico - 2008. Rio de Janeiro: Instituto Brasileiro de Geografia e Estatística; 2011.

12. World Health Organization; United Nations Child Fund. Progress on sanitation and drinking-water - 2013 update. http://www.wssinfo. org/fileadmin/user_upload/resources/JMPre port2013.pdf (acessado em 10/Mai/2015).

13. Guimarães RM, Asmus CIRF, Oliveira SA, Mazoto ML. Acesso ao saneamento básico e a internação por doença diarreica aguda: um estudo da vulnerabilidade infantil. Revista de Salud Ambiental 2013; 13:22-9.

14. Teixeira JC, Guilhermino RL. Análise da associação entre saneamento e saúde nos estados brasileiros, empregando dados secundários do banco de dados Indicadores e Dados Básicos para a Saúde - IDB 2003. Revista de Engenharia Sanitária e Ambiental 2006; 11:277-82.

15. Rasella D. Impacto do Programa Água para Todos (PAT) sobre a morbi-mortalidade por diarreia em crianças do Estado da Bahia, Brasil. Cad Saúde Pública 2013; 29:40-50.

16. Bellido JG, Barcellos C, Barbosa FS, Bastos FI. Saneamiento ambiental y mortalidad en niños menores de 5 años por enfermedades de transmisión hídrica en Brasil. Rev Panam Salud Pública 2010; 28:114-20.

17. Moura BLA, Cunha RC, Aquino R, Medina MG, Mota ELA, Macinko J, et al. Principais causas de internação por condições sensíveis à atenção primária no Brasil: uma análise por faixa etária e região. Rev Bras Saúde Matern Infant 2010; 10:583-91.

18. Govender T, Barnes JM, Pieper CH. Contribution of water pollution from inadequate sanitation and housing quality to diarrheal disease in low-cost housing settlements of Cape Town, South Africa. Am J Public Health 2011; 101:4-9. 
19. Teixeira JC, Gomes MHR, Souza JA. Associação entre cobertura por serviços de saneamento e indicadores epidemiológicos nos países da América Latina: estudos com dados secundários. Rev Panam Salud Pública 2012; 32:419-25.

20. Macinko J, Guanais FC, Souza MFM. Evaluation of impact of the Family Health Program on infant mortality in Brazil, 1990-2002. J Epidemiol Community Health 2006; 60:13-9.

21. Aquino R, Oliveira NF, Barreto ML. Impact of the Family Health Program on infant mortality in Brazilian municipalities. Am J Public Health 2009, 99:87-93.

22. Fernandes VBL, Caldeira AP, Faria AA, Rodrigues Neto JF. Internações por condições sensíveis. Rev Saúde Pública 2009; 43:928-36.

23. Barreto JOM, Nery IS, Costa MSC. Estratégia Saúde da Família e internações hospitalares em menores de 5 anos no Piauí, Brasil. Cad Saúde Pública 2012; 28:515-26.

24. Maciel AG, Caldeira AP, Dinis FJLS. Impacto da Estratégia Saúde da Família sobre o perfil de morbidade hospitalar em Minas Gerais. Saúde Debate 2014; 38:310-30.

25. Ceccon RF, Meneghel SN, Viecili PRN. Internações por condições sensíveis à atenção primária e ampliação da saúde da família no Brasil: um estudo ecológico. Rev Bras Epidemiol 2014; 17:968-77.

26. Field A. Descobrindo a estatística usando o SPSS. Porto Alegre: Editora Artmed; 2009.

27. Wagner MB, Callegari-Jacques SM. Medidas de associação em estudos epidemiológicos: risco relativo e odds ratio. J Pediatr (Rio J.) 1998; 74:247-25.

28. Prüss-Üstün A, Mathers C, Corvalán C, Woodward A. Introduction and methods: assessing the environmental burden of disease at national and local levels. Geneva: World Health Organization; 2003. (Environmental Burden of Disease Series, 1).

29. Rodrigues CG, Vormittag EMPA, Cavalcante JA, Saldiva PHN. Projeção da mortalidade e internações hospitalares na rede pública de saúde atribuíveis à poluição atmosférica no Estado de São Paulo entre 2012 e 2030. Rev Bras Estud Popul 2015; 32:489-509.

30. Freitas CU, Pereira LAA, Saldiva PHN. Vigilância dos efeitos na saúde decorrentes da poluição atmosférica: estudo de factibilidade. http:// www.cve.saude.sp.gov.br/htm/doma_vig.htm (acessado em 20/Set/2015).
31. Cançado JED. A poluição atmosférica e sua relação com a saúde humana na região canavieira de Piracicaba-SP [Tese de Doutorado]. São Paulo: Universidade de São Paulo; 2003.

32. Pokhel D, Viraraghavan T. Diarrhoeal diseases in Nepal vis-à-vis water supply and sanitation status. J Water Health 2004; 2:71-81.

33. Ganesh SK, Sitanshu SK, Animesh J. Health and environmental sanitation in India: issues for prioritizing control strategies. Indian J Occup Environ Med 2011; 15:93-6.

34. Mafra F. O impacto da atenção básica em saúde em indicadores de internação hospitalar no Brasil. Brasília: Universidade de Brasília; 2010.

35. Alves GG, Aerts D. As práticas educativas em saúde e a Estratégia Saúde da Família. Ciênc Saúde Coletiva 2001; 16:319-25.

36. Luby SP, Agboatwalla M, Painter J, Altaf A, Bilhimer WL, Hoesktra RM. Effect of intensive handwashing promotion on childhood diarrhea in high-risk communities in Pakistan: a randomized controlled trial. JAMA 2004; 291:2547-54

37. Luby SP, Halder AK, Huda T, Unicomb L, Johnston RB. The effect of handwashing at recommended times with water alone and with soap on child diarrhea in rural Bangladesh: an observational study. PLoS Med 2011; 8:e1001052.

38. Secretaria Nacional de Saneamento Ambiental, Ministério das Cidades. Sistema Nacional de Informações sobre Saneamento: diagnóstico dos serviços de água e esgotos - 2013. Brasília: Secretaria Nacional de Saneamento Ambiental, Ministério das Cidades; 2014.

39. Bühler HF, Ignotti E, Neves SMAS, Hacon SS. Análise espacial de indicadores integrados determinantes da mortalidade por diarreia aguda em crianças menores de 1 ano em regiões geográficas. Ciênc Saúde Coletiva 2014; 19:413140.

40. Szklo M, Nieto J. Epidemiología intermedia: conceptos y aplicaciones. Madrid: Ediciones Díaz de Santos; 2003. 


\section{Abstract}

The current study aimed to assess the association between socioeconomic conditions, basic sanitation, and coverage by family health teams and hospitalizations for waterborne diseases. The analysis of socioeconomic conditions and sanitation was based on an ecological study with data from the Brazilian National Household Sample Survey (PNAD) and the Brazilian Health Informatics Departament (DATASUS) database (observations for the States and Federal District) for the year 2013. Associations between family health teams and hospitalization were assessed by simple regressions, with data from DATASUS and the Department of Primary Care of Ministry of Health from 1998 to 2014. Connection to the public sewage system, low schooling, and family health team coverage were significantly associated $(p<0.05)$ with hospitalizations for waterborne diseases. Based on attributable risk analysis, for Brazil as a whole in 2013, 57,574 (16.3\%) of hospital admissions for waterborne diseases could have been avoided by adequate sewage disposal, which would also have avoided BRL 20,372,559.90 in treatment costs and 172,722 days lost to hospitalization. The results emphasize the importance of integrated sanitation policies, education, and health care that consider regional inequalities, thereby contributing to improvement of the population's health conditions.

Environmental Pollution; Basic Sanitation; Waterborne Diseases; Delivery of Health Care

\section{Resumen}

El objetivo del presente estudio fue evaluar la asociación entre condiciones socioeconómicas, de saneamiento básico y de cobertura por equipos de salud de la familia sobre los internamientos por enfermedades de transmisión hídrica en Brasil. El análisis de las condiciones socioeconómicas $y$ de saneamiento se realizó mediante un estudio ecológico, con datos de la Encuesta Nacional por Muestra de Domicilios (PNAD) y del Sistema de Información del Sistema Único de Salud (DATASUS) -observaciones de los Estados y Districto Federal-durante el año 2013. La relación de los equipos de salud de la familia con los internamientos se evaluó gracias a la estimación de regresión simple, con los datos recogidos en el DATASUS y el Departamento de Atención Básica del Ministerio de la Salud, para el período de 1998 a 2014. La existencia de desagües para los residuos, mediante una red general de alcantarillado, la baja escolaridad y la cobertura por equipos de salud de la familia influenciaron con significancia estadística $(p<0,05)$ los internamientos evaluados. Por el análisis del riesgo atribuible, se estimó que en Brasil, tomando como base los datos de 2013, 57.574 (16,3\%) de los internamientos por enfermedades seleccionadas podrían haber sido evitados, si las condiciones del alcantarillado fueran las adecuadas. Se evitarían incluso los gastos con su tratamiento, calculado en BRL 20.372.559,90, y los dias perdidos con los internamientos en 172.722. Se sugiere que la adopción integrada de politicas de saneamiento, educación y asistencia a la salud, que consideren las desigualdades regionales, contribuiría a la mejoría de las condiciones de salud de la población.

Contaminación Ambiental; Saneamiento Básico; Enfermedades Transmitidas por el Agua;

Prestación de Atención de Salud
Recebido em 01/Fev/2016

Versão final reapresentada em 01/Fev/2017

Aprovado em 25/Abr/2017 